\title{
POLARIZATION OF PROMPT GAMMA-RAY BURST EMISSION: EVIDENCE FOR ELECTROMAGNETICALLY DOMINATED OUTFLOW
}

\author{
M. Lyutikov, ${ }^{1,2}$ V. I. PARIEv, ${ }^{3,4}$ AND R. D. BlandForD ${ }^{5}$ \\ Received 2003 May 21; accepted 2003 July 17
}

\begin{abstract}
Observations by the RHESSI satellite of the large polarization of the prompt $\gamma$-ray emission from $\gamma$-ray burst (GRB) 021206 imply that the magnetic field coherence scale is larger than the size of the visible emitting region, $\sim R / \Gamma$, where $R$ is the radius of the flow and $\Gamma$ is the associated Lorentz factor. Such fields cannot be generated in a causally disconnected, hydrodynamically dominated outflow. Electromagnetic models of GRBs, in which large-scale, dynamically dominant, magnetic fields are present in the outflow from the very beginning, provide a natural explanation of this large reported linear polarization. We derive the Stokes parameters of the synchrotron emission of a relativistically moving plasma with a given magnetic field configuration and calculate the pulse-averaged polarization fraction of the emission from a relativistically expanding shell carrying a global toroidal magnetic field. For viewing angles larger than $1 / \Gamma$, the observed patch of the emitting shell has an almost homogeneous magnetic field, producing a large fractional polarization ( $56 \%$ for a power-law energy distribution of relativistic particles, $\left.d n / d \epsilon \propto \epsilon^{-3}\right)$. The maximum polarization is smaller than the theoretical upper limit for a stationary plasma in a uniform magnetic field because of relativistic kinematic effects.
\end{abstract}

Subject headings: gamma rays: bursts — MHD — polarization

\section{INTRODUCTION}

The origin of the magnetic fields in $\gamma$-ray bursts (GRBs) is one of the central unresolved issues of astrophysics. In the standard fireball scenario (e.g., Piran 1999; Mészáros 2002 and references therein), the magnetic field does not play any dynamical role. The near-equipartition field invoked in the emission region is assumed to be generated locally at relativistic shocks by plasma instabilities (e.g., Medvedev \& Loeb 1999). Initially, the spatial scale of such fields is microscopically small, of the order of the ion skin depth, $\delta \sim c / \omega_{p, i}\left(\omega_{p, i}\right.$ is the ion plasma frequency). Although the typical scale of magnetic field fluctuations may grow because of inverse cascade even in the unlikely case that such growth proceeds at the speed of light, the resulting polarization is expected to be smaller than $10 \%$ (e.g., Gruzinov \& Waxman 1999).

The recent detection by the RHESSI satellite of large polarization in prompt $\gamma$-ray emission (Coburn \& Boggs 2003) places severe constraints on GRB models. It implies that the magnetic field coherence scale is larger than the size of the visible emitting region, $\sim R / \Gamma$, where $R$ is the distance from the center and $\Gamma$ is the bulk Lorentz factor of the relativistically expanding emission region. Such fields cannot be generated in a hydrodynamically dominated outflow, which is causally disconnected on large scales. Thus, the largescale magnetic fields should be present in the outflow from

\footnotetext{
${ }^{1}$ Physics Department, McGill University, 3600 rue University, Montreal, QC H3A 2T8, Canada; lyutikov@physics.mcgill.ca.

2 Canadian Institute for Theoretical Astrophysics, 60 St. George Street, Toronto, ON M5S 3H8, Canada.

${ }^{3}$ Department of Physics and Astronomy, University of Rochester, Rochester, NY 14627.

${ }^{4}$ Lebedev Physical Institute, Leninsky Prospect 53, Moscow 119991, Russia.

5 Theoretical Astrophysics, California Institute of Technology, Pasadena, CA 91125.
}

the very beginning. In fact, as we argue below, such fields must be dynamically dominant, carrying most of the energy of the outflow.

Building on earlier models of electromagnetic explosions (e.g., Usov 1992; Thompson 1994; Smolsky \& Usov 1996; Mészáros \& Rees 1997), Lyutikov \& Blandford (2002, 2003) developed an electromagnetic model of GRBs that assumes that a rotating, relativistic, stellar-mass progenitor (e.g., "millisecond magnetar"; Usov 1992) loses much of its spin energy in the form of an electromagnetically dominated outflow. A stellar-mass relativistic progenitor is born with an angular velocity $\Omega \sim 10^{4} \mathrm{~s}^{-1}$ and a dynamo-generated magnetic field of $B_{s} \sim 3 \times 10^{14} \mathrm{G}$. Then, the total rotational energy, $E \sim I \Omega^{2} / 2 \sim 5 \times 10^{52}$ ergs (for a $1.4 M_{\odot}$ object), is available to power GRB bursts, while the dipole spin-down luminosity, $L_{P} \simeq B_{s}^{2} r_{s}^{6} \Omega^{4} / c^{3} \simeq 10^{49} \mathrm{ergs} \mathrm{s}^{-1}$, is about the luminosity of cosmological GRBs. In this model the energy to power the GRBs comes eventually from the rotational energy of the progenitor. It is first converted into magnetic energy by the dynamo action of the unipolar inductor, propagated in the form of a Poynting-flux-dominated flow, and then dissipated at large distances from the source.

A rapidly spinning magnetar with a complicated field structure will form a relativistic outflow. We suggest that the magnetic field in the wind quickly rearranges to become predominantly axisymmetric. There is a good precedent for this behavior in Ulysses observations of the quiet solar wind (McComas et al. 2000), which reveal that, despite the complexity of the measured surface magnetic field, the field in the solar wind quickly rearranges to form a good approximation to a Parker (1960) spiral. The situation in the far field will then resemble that first analyzed by Goldreich \& Julian (1969), and the characteristic scale length in the far field will be the cylindrical radius from the polar axis, rather than the wavelength.

During the relativistic expansion, most of the magnetic energy carried by the axisymmetric toroidal magnetic fields 
is concentrated in a thin shell with thickness $\Delta r \sim c t_{s} \sim$ $3 \times 10^{12} \mathrm{~cm}$ inside a contact discontinuity separating the ejecta from the shocked circumstellar material. At the contact discontinuity, the toroidal magnetic field balances the ram pressure of the circumstellar material: $B_{\phi} \sim$ $4 \Gamma^{2}\left(\pi \rho_{\mathrm{ext}} c^{2}\right)^{1 / 2}$. Both $B_{\phi}$ and $\Gamma$ depend on the angle between a given point on the shell and the polar axis, defined as the axis of rotation of the progenitor. This results in a nonspherical, relativistic expansion of the shell. In particular, for laterally balanced expansion, $\Gamma \sim 1 / \sin \theta$. The currentcarrying shell becomes unstable because of the development of current-driven instabilities at a radius of $\sim 10^{16} \mathrm{~cm}$. This leads to the acceleration of pairs that emit $\gamma$-rays by synchrotron radiation.

A distinctive feature of the electromagnetic model is that the causal connection is better than in the hydrodynamic models. Initially, close to the central source the subsonic flow is fully causally connected. As the flow is accelerated by magnetic (and partially by pressure) forces, it becomes supersonic, strongly relativistic, and causally disconnected over small polar angles $\Delta \theta \sim 1 / \Gamma$. Later, magnetically dominated flows quickly reestablish causal contact over large polar angles and become fully causally connected again after a time $t_{c} \sim t_{s} \Gamma^{2}$, where $t_{s} \sim 100 \mathrm{~s}$ is the source activity lifetime. This is drastically different from hydrodynamic flows, which remain causally disconnected over polar angles larger than $1 / \Gamma$. Thus, during expansion the causal behavior of the flow resembles the behavior of cosmic fluctuations during inflation: as the flow expands, angular scales $\sim \Gamma^{-1}$ "enter the horizon," reestablishing causal contact that was lost during acceleration.

To illustrate this behavior, consider the propagation of a sound-type disturbance emitted by a point source located on the relativistically moving shell at radius $R_{\mathrm{em}}$. Let the typical signal speed in the plasma rest frame be $\beta_{s}$. In Appendix A we show that for sub-Alfvénic ejecta (magnetically dominated flows can be strongly relativistic, but still sub-Alfvénic!), a relativistically expanding shell reestablishes a causal contact over the visible patch of $1 / \Gamma$ in just one dynamical timescale (after doubling in radius). If the ratio of the magnetic to particle energy density in the cold, magnetized plasma is $\sigma=u_{B} / u_{p} \gg 1$ (Kennel \& Coroniti 1984), then the Alfvén (and fast magnetosound) velocity is $\beta_{\mathrm{A}}=c[\sigma /(1+\sigma)]^{1 / 2}$. The requirement that the expansion velocity be sub-Alfvénic then implies that $c \Gamma \leq u_{\mathrm{A}}$, or $\sigma \gg 1$. Therefore, the condition that magnetic fields have a coherence scale larger than $R / \Gamma$ requires that the magnetic fields be energetically dominant in the flow.

Hydrodynamic (e.g., fireballs [Piran 1999] or external shocks [Dermer 2002] with $\sigma \ll 1$ ) or hydromagnetic models $(\sigma \sim 1$; e.g., Spruit, Daigne, \& Drenkhahn 2001; Drenkhahn \& Spruit 2002; Vlahakis \& Königl 2003) could also have a large-scale ordered magnetic field (cannonballs [see, e.g., Dar 2003] need to rely on energetically inefficient Compton scattering and strong flow inhomogeneities on the angular scale $\sim 1 / \Gamma$; Coburn \& Boggs 2003; $\S 3$; we consider this a prohibitively tight constraint). In all cases, the whole outflow is in causal contact close to the source and may have a large-scale magnetic field that will be carried with the flow. In hydrodynamically dominated models, after the causal contact is lost, different parts of the flow cannot communicate and thus will evolve differently, depending on the local conditions. Only under strict homogeneity of the surrounding medium and of the ejecta will the two causally discon- nected parts of the flow have similar properties. On the other hand, since magnetically dominated outflows can quickly communicate information (e.g., magnetic pressure) over large polar angles, they can have quasi-homogeneous properties despite possible inhomogeneities in the circumstellar medium and in the ejecta.

The assumption of an electromagnetically dominated flow must eventually break down, since the ejecta need to dissipate magnetic energy to produce high-energy emission. In the emission region, the plasma is expected to be close to equipartition, since the magnetic field is dissipated to accelerate electrons (generally, equipartition is needed for effective emission). However, unlike the hydrodynamic models in which equipartition is reached by amplifying weak magnetic fields at the shocks, in the electromagnetically dominated model, the equipartition is reached by dissipation of the initially dominant magnetic field, as, for example, happens in solar flares.

In this paper we calculate the Stokes parameters for the prompt GRB emission emerging in the electromagnetic model as a function of the viewing angle (the angle between the line of sight and the polar axis of the flow). We assume that the magnetic field in the emission region is dominated by the toroidal field and is concentrated in a thin shell $\Delta R$ near the surface of the shell, expanding with Lorentz factor $\Gamma(\theta)$. Synchrotron emission is produced by an isotropic population of relativistic electrons with a power-law distribution in energy. In the present work we calculate the Stokes parameters averaged over the duration of the GRB pulse, deferring the time-dependent calculations to a later work. One expects that the polarization fraction will be maximal in the beginning of the pulse, slightly decreasing toward the end as larger emitting volumes become visible. We set the speed of light to unity, $c=1$, in all the expressions to follow.

\section{CALCULATION OF STOKES PARAMETERS}

Consider a quasi-spherical thin emitting shell (Fig. 1) viewed by an observer. Below we denote all quantities measured in the local frame comoving with an emitting elementary volume with primes, while unprimed notations refer to the quantities measured in the explosion frame. Let $r, \theta$, and $\phi$ be spherical coordinates in a coordinate system with the origin at the center of the shell and $x, y$, and $z$ be rectangular coordinates with the origin at the center of the shell. The symmetry axis of the shell is the $z$-axis. The toroidal magnetic field in the shell is in the $\phi$-direction. The observer is located in the $x-z$ plane. The components of all vectors written below are the components with respect to the rectangular coordinate system $(x, y, z)$. The shell expands quasi-spherically with an angle-dependent Lorentz factor $\Gamma(\theta)$. An element of the shell moving radially with velocity $\boldsymbol{v}=\beta\{\sin \theta \cos \phi, \sin \theta \sin \phi, \cos \theta\}$ emits a burst of synchrotron radiation in the direction of unit vector $\boldsymbol{n}=\left\{\sin \theta_{\mathrm{ob}}, 0, \cos \theta_{\mathrm{ob}}\right\}$ when viewed in the observer frame.

Several key ingredients need to be taken into account (e.g., Cocke \& Holm 1972; Blandford \& Königl 1979; Bjornsson 1982; Ginzburg 1989). First, the synchrotron emissivity depends on the direction between the emitted photon and the magnetic field in the plasma rest frame. Second, as the emission is boosted by the relativistic motion of the shell, the position angle of the linear polarization 


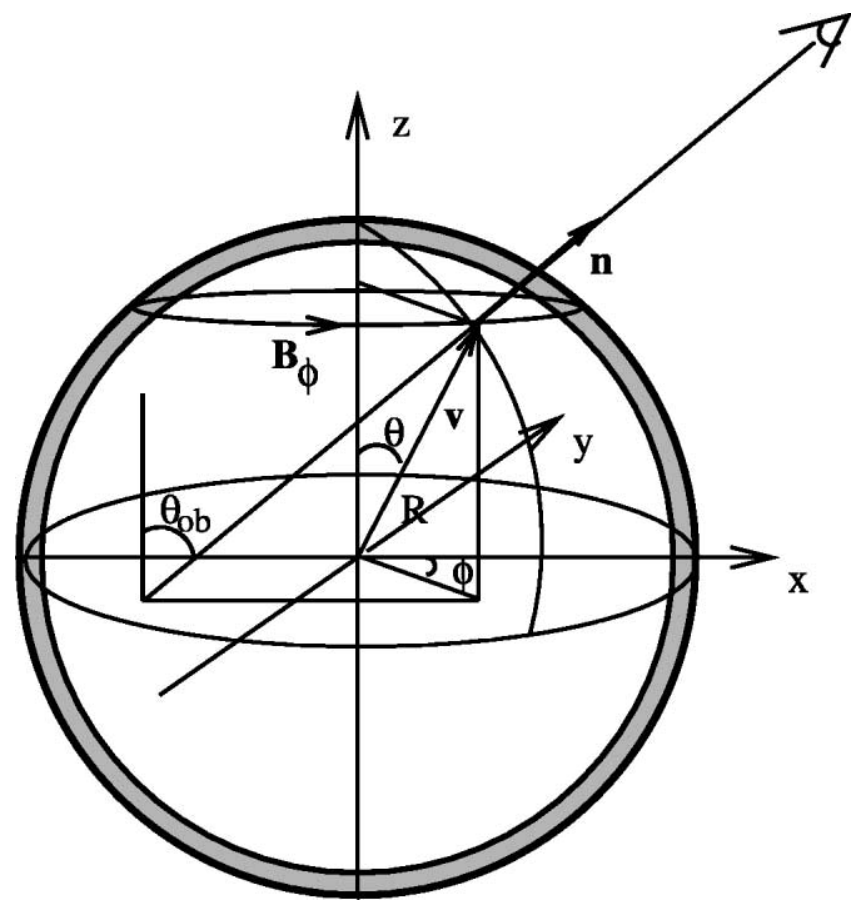

FIG. 1.-Geometry of the model. A narrow shell $\Delta r \sim t_{s} c \sim 3 \times 10^{12} \mathrm{~cm}$, dominated by a toroidal magnetic field, expands quasi-spherically with angle-dependent velocity $\boldsymbol{v}(\theta)$. The observer is located at an angle $\theta_{\mathrm{ob}}$ with respect to the polar axis.

rotates in the $\boldsymbol{n}$ - $\boldsymbol{v}$ plane. ${ }^{6}$ The fractional polarization emitted by each element remains the same, but the direction of the polarization vector of the radiation emitted by different elements within a visible shell is rotated by different amounts. This leads to effective depolarization of the total emission. The theoretical maximum polarization fraction for a homogeneous field can be achieved only for a uniform plane-parallel velocity field. Third, integration along the line of sight (and over the emitting solid angle for unresolved sources) is better carried out in the laboratory frame, in order to take correct account of the arrival times of the photons.

We assume that the distribution function of the emitting particles in the frame comoving with an element of the shell is isotropic in momentum and is a power law in energy:

$$
d n=K_{e} \epsilon^{-p} d \epsilon d V d \Omega_{p} .
$$

Here $d n$ is the number of particles in the energy interval $(\epsilon$, $\epsilon+d \epsilon), d V$ is the elementary volume, $d \Omega_{p}$ is the elementary solid angle in the direction of the particle momentum $\boldsymbol{p}$, $K_{e}=K_{e}(r)$, and $p=$ constant.

In this paper we are interested in the polarization structure of the time-integrated pulse of the emission and not in its temporal properties. Hence, for simplicity we approximate the $\gamma$-ray emissivity of the shell as a flash at some time $t_{0}$ in the explosion frame, lasting for $\Delta t \ll \Delta R / c$, where $\Delta R \ll R_{0}$ is the thickness of the shell at the moment $t_{0}$ and $R_{0}$ is the radius of the shell at the moment $t_{0}$. More complicated emission profiles can be easily accommodated. In addition, we integrate over the observer time to get an

${ }^{6}$ This effect has been missed by all previous calculations of GRB polarization. average polarization of the pulse, deferring time-dependent calculations to a later work.

We also assume that the emission is optically thin and neglect possible plasma propagation effects (e.g., depolarization of radiation due to internal Faraday rotation by low-energy electrons). Since the emitting particles are ultrarelativistic and we neglect conversion of linear to circular polarization in the plasma, we do not have circular polarization in our model (Stokes $V=0$ ). We also neglect a possible tangled component of the magnetic field present in the emission region. We assume that the emission originates in a geometrically thin layer $\Delta R \ll R$ with the thickness $\Delta R$ independent of $\theta$ and neglect variation of the magnetic field and velocity across the layer. Given these assumptions, our estimates provide an upper limit on the possible polarization.

The time-integrated Stokes parameters are calculated in Appendix B (eqs. [B7]). Because of the cylindrical symmetry of the model, the Stokes parameter $\bar{U}$ integrates to zero, so that the observed averaged polarization fraction is

$$
\begin{aligned}
\Pi= & \frac{|\bar{Q}|}{\bar{I}}=\frac{p+1}{p+7 / 3} \\
& \times \frac{\int \sin \theta d \theta d \phi \mathscr{D}^{2+(p-1) / 2}\left|B^{\prime} \sin \chi^{\prime}\right|^{(p+1) / 2} \cos 2 \tilde{\chi}}{\int \sin \theta d \theta d \phi \mathscr{D}^{2+(p-1) / 2}\left|B^{\prime} \sin \chi^{\prime}\right|^{(p+1) / 2}} .
\end{aligned}
$$

Here $B^{\prime}$ is the magnitude of the magnetic field $\boldsymbol{B}^{\prime}$ in the frame of an element of the shell, $\chi^{\prime}$ is the angle that the line of sight in the frame of an element of the shell, $\boldsymbol{n}^{\prime}$, makes with the magnetic field $\boldsymbol{B}^{\prime}$, and $\tilde{\chi}$ is the position angle of the electric field vector in the observer plane of the sky measured from some reference direction. The Doppler boosting factor is $\mathscr{D}=1 / \Gamma(1-\boldsymbol{n} \cdot \boldsymbol{v})$. For a toroidal magnetic field $\bar{Q}>0$, so that the observed polarization vector is always along the projection of the flow axis on the plane of the sky.

The evaluation of the different quantities in equation (2) is an involved exercise in Lorentz transformations. We assume that, in the shell frame, the magnetic field is purely toroidal,

$$
\boldsymbol{B}^{\prime}=b_{\phi}(r, \theta) \hat{\boldsymbol{B}}^{\prime}=b_{\phi}\{-\sin \phi, \cos \phi, 0\},
$$

where $\hat{\boldsymbol{B}}^{\prime}$ is the unit vector along $\boldsymbol{B}^{\prime}$ in the radiating element frame and $b_{\phi}$ is the magnitude of the field. A photon propagating along the unit vector $\boldsymbol{n}$ in the explosion frame is emitted along the direction of the unit vector $\boldsymbol{n}^{\prime}$ in the radiating element frame:

$$
\boldsymbol{n}^{\prime}=\frac{\boldsymbol{n}+\Gamma \boldsymbol{v}\{[\Gamma /(\Gamma+1)](\boldsymbol{n} \cdot \boldsymbol{v})-1\}}{\Gamma[1-(\boldsymbol{n} \cdot \boldsymbol{v})]} .
$$

Note that $\boldsymbol{n}^{\prime}, \boldsymbol{n}$, and $\boldsymbol{v}$ lie in the same plane. The angle $\chi^{\prime}$ between the photon and magnetic field in the radiating element frame is

$$
\begin{aligned}
\cos \chi^{\prime} & =\hat{\boldsymbol{B}}^{\prime} \cdot \boldsymbol{n}^{\prime} \\
& =\frac{\left(\hat{\boldsymbol{B}}^{\prime} \cdot \boldsymbol{n}\right)+\Gamma\left(\hat{\boldsymbol{B}}^{\prime} \cdot \boldsymbol{v}\right)\{[\Gamma /(\Gamma+1)](\boldsymbol{n} \cdot \boldsymbol{v})-1\}}{\Gamma[1-(\boldsymbol{n} \cdot \boldsymbol{v})]},
\end{aligned}
$$

which gives

$$
\sin ^{2} \chi^{\prime}=1-\frac{\sin ^{2} \phi \sin \theta_{\mathrm{ob}}^{2}}{\Gamma^{2}(1-\beta \mu)^{2}},
$$

where $\mu=\cos \theta \cos \theta_{\mathrm{ob}}+\sin \theta \sin \theta_{\mathrm{ob}} \cos \phi$. 
We also need to evaluate the angle $\tilde{\chi}$ between a given direction in the observer plane and the polarization vector. This is not trivial, since the polarization vector emitted by each element will experience rotation during Lorentz transformation from the shell frame to the laboratory frame (Cocke \& Holm 1972; Blandford \& Königl 1979). The rotation of the polarization vector is due to the rotation of the wavevector in the plane containing vectors $\boldsymbol{n}, \boldsymbol{n}^{\prime}$, and $\boldsymbol{v}$ and the requirement that the electric field of the wave remain orthogonal to the wavevector. Since the wavevectors of the emitted waves experience rotation by angles of the order of unity, this effect would lead to the effective depolarization of emission from a medium with a nonuniform velocity field, even with a homogeneous magnetic field. In Appendix $\mathrm{C}$ we derive the general relations for the Lorentz transformation of the polarization vector.

We choose to measure the angle $\tilde{\chi}$ clockwise from the direction parallel to the projection of the axis of the flow on the plane of the sky. The unit vector in this direction is $\left\{-\cos \theta_{\mathrm{ob}}, 0, \sin \theta_{\mathrm{ob}}\right\}$. We find (Appendix C)

$$
\begin{aligned}
& \cos \tilde{\chi}=\frac{(1-\beta \mu) \cos \phi-\beta \sin \theta \sin \theta_{\mathrm{ob}} \sin ^{2} \phi}{\sqrt{(1-\beta \mu)^{2}-\sin ^{2} \theta_{\mathrm{ob}} \sin ^{2} \phi / \Gamma^{2}}}, \\
& \sin \tilde{\chi}=\frac{\sin \phi\left(\beta \cos \theta-\cos \theta_{\mathrm{ob}}\right)}{\sqrt{(1-\beta \mu)^{2}-\sin ^{2} \theta_{\mathrm{ob}} \sin ^{2} \phi / \Gamma^{2}}} .
\end{aligned}
$$

In the ultrarelativistic limit, $\Gamma \gg 1$, the general relations simplify, and it becomes possible to determine analytically the maximum polarization fraction for a given velocity field in the limit $\theta_{\mathrm{ob}} \Gamma \gg 1$ (Appendix D). For $p=3$ we find $\Pi=9 / 16$, in excellent agreement with numerical calculations.

In Figure 2 we plot the map of the polarized emissivity from the shell moving with a constant $\Gamma$ and with constant $b_{\phi}$ as seen by an observer on the plane of the sky. Here $l$ and $s$ are rectangular coordinates on the plane of the sky centered at the projection of the center of the shell. The $s$-axis is
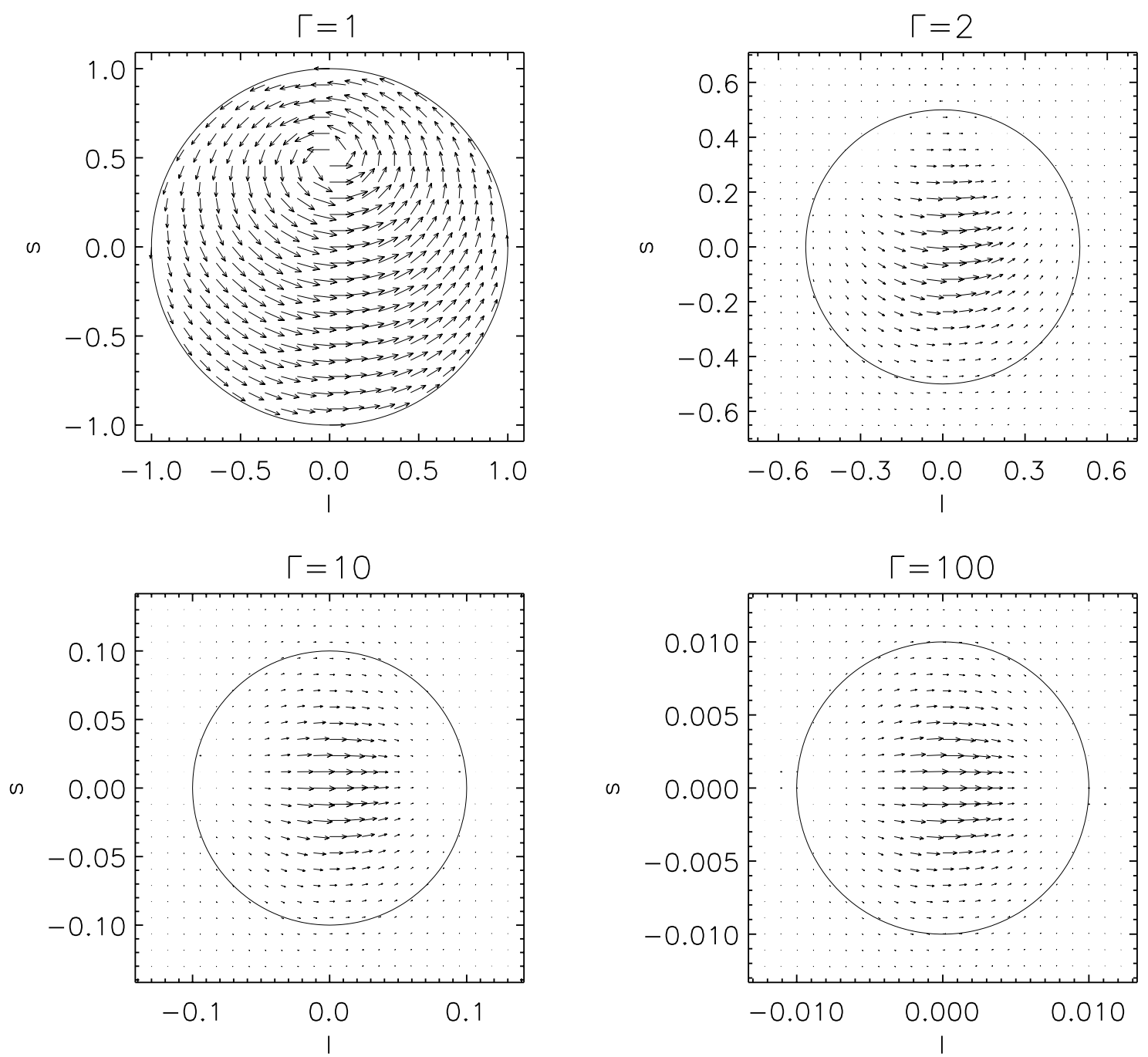

FIG. 2.-Polarization map of the emission from the GRB on the observer plane of the sky in rectangular coordinates $l$ and $s$. The $s$-axis is directed parallel to the projection of the axis of the shell on the sky. The observer line of sight makes a $30^{\circ}$ angle with the axis of the shell, $\theta_{\mathrm{ob}}=30^{\circ}$. Plots are made for four different values of $\Gamma$ and $p=3$. The solid circles have radii $1 / \Gamma$. Since the intensity of the radiation is highly peaked in an area of size $\sim 1 / \Gamma$, we zoomed in on this area in the plots with $\Gamma \gg 1$. 
directed parallel to the projection of the axis of the shell on the sky, and $l$ and $s$ are normalized such that the projection of the shell radius, $R_{s}$, is a circle of radius 1 in the $l-s$ plane. Thus,

$$
\begin{gathered}
l=\frac{1}{R_{s}} \boldsymbol{l} \cdot \boldsymbol{r}=\sin \theta \cos \phi, \\
s=-\frac{1}{R_{s}} \boldsymbol{r} \cdot(\boldsymbol{l} \times \boldsymbol{n})=-\cos \theta_{\mathrm{ob}} \sin \theta \cos \phi+\sin \theta_{\mathrm{ob}} \cos \theta,
\end{gathered}
$$

where $\boldsymbol{l}=\{0,1,0\}$ is the unit vector along $l$. The arrows on the plots in Figure 2 are directed perpendicular to the unit vector in the direction of the electric field of the wave, $\hat{\boldsymbol{e}}$, so that in the nonrelativistic limit, $\Gamma \rightarrow 1$, the arrows are aligned with the magnetic field $\boldsymbol{B}$. The length of the arrows is proportional to the synchrotron emissivity from the unit volume, i.e., to the expression under the integral for $\bar{I}$ in equations (B7). The actual observed intensity is modified by a geometric factor proportional to the path of the ray inside the volume of the shell. For $R_{s}-r \gg \Delta R$ the geometric factor is $1 / \mu$. For $\Gamma \gg 1$ Doppler boosting leads to a small effective emitting area of the shell: $l \leq 1 / \Gamma$ and $s \leq 1 / \Gamma$. The relativistic swing of the polarization vector is also clearly visible in Figure 2. Each patch of the shell emits radiation with the same polarization degree, $\Pi_{\max }=(p+1) /$ $(p+7 / 3)$. Because of summation over areas of the shell with different directions of $\tilde{\chi}$, the resulting polarization degree becomes smaller than $\Pi_{\max }$.

We are now in a position to estimate the polarization fraction in equation (2), integrating the Stokes parameters (eq. [B7]) over an expanding relativistic shell. The results for $\Pi$ are shown in Figure 3. The parameter $\bar{Q}$ is zero for $\theta_{\mathrm{ob}}=0$ and is small for $\theta_{\mathrm{ob}}<1 / \Gamma$, because the polar axis falls within the visible patch in this case. The magnetic field

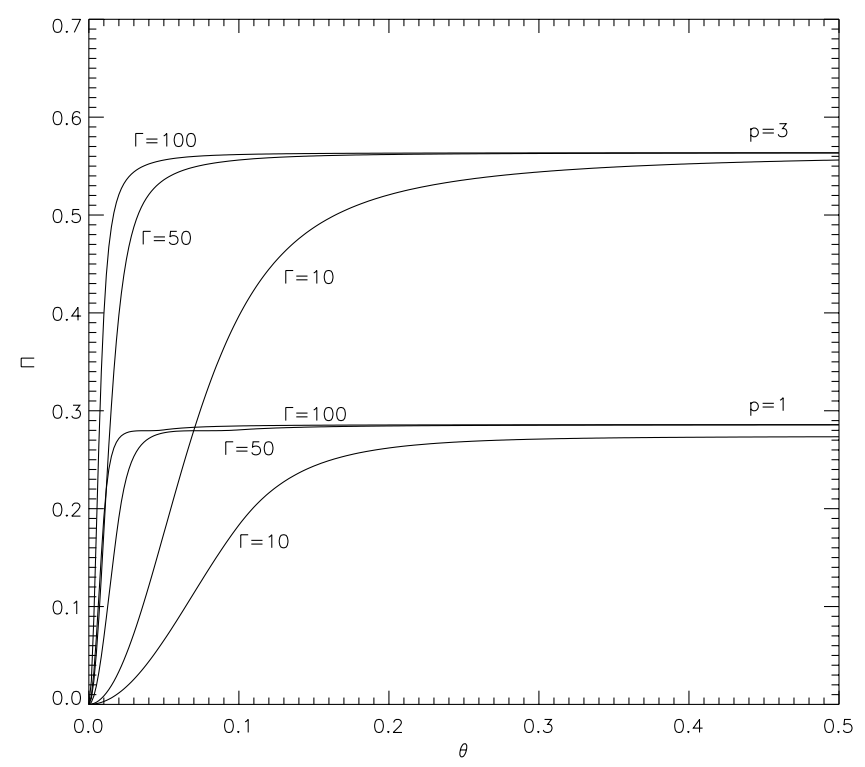

FIG. 3.-Dependence of the polarization fraction $\Pi=Q / I$ on the viewing angle $\theta_{\mathrm{ob}}$ for different Lorentz factors $\Gamma$, for isotropic expansion $[\Gamma(\theta)=$ const $=10,50$, and 100 ; right to left $]$ and a power-law particle distribution: $d n / d \epsilon=\epsilon^{-p}(p=3$ : top curves; $p=1$ : bottom curves; for $p=2$ the asymptotic value is $43 \%$ ). At $\theta_{\mathrm{ob}}=0$, the polarization is zero, growing to large values when $\theta_{\mathrm{ob}}>1 / \Gamma$. The depolarization of emission due to the differential rotation of the position angle of the linear polarization in the $\boldsymbol{n} \boldsymbol{v} \boldsymbol{v}$ plane reduces the maximum possible polarization fraction to below the theoretical limit for a homogeneous field. changes its direction within the visible patch, and the resulting polarization is reduced. The degree of polarization reaches a limiting value of tens of percent when the observation angle is larger than $1 / \Gamma$.

\section{DISCUSSION}

Large-scale ordered magnetic fields produced at the central source provide a simple explanation of the recent observations of highly polarized GRB prompt emission by the RHESSI satellite (Coburn \& Boggs 2003). In order to retain the coherence of the magnetic field on scales larger than the visible patch, $\sim R / \Gamma$, the ejecta must be electromagnetically dominated. The electromagnetic model suggested by Lyutikov \& Blandford $(2002,2003)$ provides a solution to the puzzle of how to produce large coherent magnetic fields and how to launch a blast wave that extends over an angular scale $\gg \Gamma^{-1}$, where the individual parts are out of causal contact. In the electromagnetic model, the magnetic fields are present in the outflow from the very beginning, and the energy is transferred to the blast wave by a magnetic shell that is causally connected at the end of the coasting phase.

To prove this point, we first found general relations for the transformation of the polarization direction of the synchrotron emission produced by a relativistically moving source with a given magnetic field structure and calculated the Stokes parameters for the time-averaged synchrotron emission for the particular case of a relativistically expanding shell containing toroidal magnetic fields. We find that for observing angles satisfying $\theta_{\mathrm{ob}} \geq 1 / \Gamma$, a large polarization fraction $\Pi \sim 60 \%$ may be observed (the actual spectrum was not measured for GRB 021206). The position angle of the polarization is fixed by the projection of the progenitor axis on the plane of the sky and thus should not change during the burst.

Another potential source of polarization could be Compton scattering of unpolarized $\gamma$-rays. If unpolarized $\gamma$-rays are initially beamed into a small-angle jet and are scattered by the surrounding gas, then polarized scattered $\gamma$-rays would be distributed nearly isotropically. This would require a much higher energy in the initial $\gamma$-ray jet than the energy necessary for the narrowly beamed jet to be observed directly (Coburn \& Boggs 2003), thus putting a much tougher requirement on the total energy budget of the GRB. Compton scattering by a relativistically moving wideangle envelope could also occur. In this case, the kinematics of the scattering would be similar to the kinematics of the synchrotron emission considered in the present work. Therefore, the energetic requirements would also be similar to the synchrotron mechanism. However, Comptonscattered photons do not have a preferred direction of polarization, which is set by the large-scale magnetic field in the synchrotron case. The net polarization of the scattered photons from the uniform spherical shell averages to zero. High polarization can be observed only if the shell parameters ( $\Gamma$ or the electron density) vary significantly on the angular scale $\sim 1 / \Gamma$. We are coming back to a highly collimated flow. Therefore, we conclude that Compton scattering cannot account for the high degree of polarization of $\gamma$-rays emerging from a wide-angle expanding flow. We note that the synchrotron mechanism results in the electric vector of the polarized emission being directed parallel to the axis of the flow, while the scattered $\gamma$-rays would be polarized in the direction perpendicular to the axis of flow. 
Several natural correlations between GRB polarization and other parameters follow from the model and can be tested with future observations. First, the polarization fraction should decrease from the beginning to the end of the pulse as larger areas of the emitting shell become visible to the observer. Second, the maximum amount of polarization should be related to the spectrum of the emitting particles, being higher for softer spectra. This points to a possible correlation between the amount of polarization and the hardness of the spectrum.

Our treatment of the prompt emission can also be related to the polarization of afterglows. If the field from the magnetic shell were mixed in with the shocked circumstellar material (similar to the so-called flux transfer events at the day side of the Earth magnetosphere), then a comparably large fractional polarization could be observed in afterglows as well. In addition, since the preferred direction of polarization is always aligned with the flow axis, the position angle should not be changing through the afterglow (if polarization is observed in both the prompt and afterglow emission, the position angle should be the same). In addition, polarization should not be related to the "jet-break" moment. This is in stark contrast to the jet model, in which polarization is seen only near the jet-break times, and the position angle is predicted to experience a flip during the jet break (Sari 1999). For the same reason, a large average polarization cannot be due to a particular viewing geometry, as suggested by Waxman (2003). Current polarization data show that in virtually all cases, the position angle remains constant (Covino et al. 2003a, 2003b; Barth et al. 2003; Bersier et al. 2003; see, however, Rol et al. 2003), while the amount of polarization does not show any correlation with the jet break. This is consistent with the presence of large-scale ordered magnetic fields in the afterglows. (A model of Rossi et al. 2002 of structured jets also predicts a constant position angle, but since no large-scale magnetic field is assumed, the polarization features are still related to the jet-break times.)

In our calculations we have neglected a random component of the magnetic field that must be present in the emission region. Lyutikov \& Blandford $(2002,2003)$ suggested that $\gamma$-ray-emitting electrons are accelerated by current instabilities, somewhat similar to solar flares. The development of current instabilities should be accompanied by the dissipation of magnetic fields and destruction of the magnetic flux. These will generally add a random component to the ordered magnetic field and will lead to a decrease in polarization (Korchakov \& Syrovatskii 1962). The corresponding calculations are in progress.
An alternative model of GRBs that can feasibly give large-scale magnetic fields in the prompt emission region is the plerion model (Königl \& Granot 2002; Inoue, Guetta, \& Pacini 2003; see also Lyutikov 2002), which initially was suggested for afterglows but can also be extended to include the prompt emission from an external shock wave (Dermer 2002). In this case, the large-scale equipartition magnetic fields are created ahead of the expanding GRB ejecta by the preceding explosion of the supranova (Vietri \& Stella 1999). Still, this type of model faces a similar causality/efficiency problem: if the plerion plasma is only at equipartition, $\sigma \sim 1$, it can be expected to be inhomogeneous on the $R / \Gamma$ scale; if it is strongly magnetized, $\sigma \gg 1$, then the shocks will be only weakly dissipative.

Implications of the RHESSI results, that GRB flows are electromagnetically driven, may provide an important clue to the dynamics of other astrophysical sources, such as pulsars, (micro)quasars, and active galactic nuclei (AGNs). It is quite plausible that all these sources produce ultrarelativistic, magnetically dominated outflows with a low baryon density (Blandford 2002). The flow evolution in all these systems may proceed in a similar way. Energy, transported primarily by magnetic fields, is dissipated far away from the source because of the development of current instabilities. Particles are accelerated in localized current sheets by direct current electric fields and/or electromagnetic turbulence, producing bright knots (in AGNs) and a variety of bright spots in pulsar jets, best observed in the Crab.

Note added in manuscript.-After the submission of the paper, additional observational details of the burst GRB 021206 became available. The spectral index of the burst is $\alpha \sim 0.6$; the corresponding particle index is $p=2.2$ (W. Hajdas 2003, private communication). The maximum polarization in our model is then $\sim 45 \%$. In addition, there were several theoretical developments. A higher polarization (up to $100 \%$ ) can be achieved if the particle distribution is not isotropic (D. Lazzati 2003, private communication). Granot (2003) has performed polarization calculations similar to ours, taking into account a random component of the magnetic field. He reached a similar conclusion that it is substantially easier to produce the polarization observed in GRB 021206 from an ordered magnetic field. A small discrepancy between the results is explained by the difference in the duration of the emission: while we assumed that the shell emits during the time $\Delta t \ll \Delta r$, Granot (2003) assumed that the emission is more prolonged: $\Delta t \gg \Delta r \Gamma^{2}$.

V. P. acknowledges support from Department of Energy grant DE-FG02-00ER54600.

\section{APPENDIX A}

\section{CAUSAL STRUCTURE OF RELATIVISTIC MAGNETIZED OUTFLOWS}

In this appendix we consider the causal structure of a relativistically expanding magnetized shell. We wish to answer the question: If the surface of a relativistically expanding magnetized shell is perturbed at a given radius $R_{\mathrm{em}}$ and zero polar angle, which points on the surface of the shell will be affected after time $t$ ?

Consider the propagation of a sound-type disturbance emitted by a point source located on the surface of a relativistically moving shell at radius $R_{\mathrm{em}}$. Let the signal speed in the plasma rest frame be $\beta_{s}$. For simplicity, we assume that the shell is moving with a constant velocity. If in the plasma frame a wave is emitted in the direction $\theta_{\mathrm{em}}$ with respect to the flow velocity, ${ }^{7}$

\footnotetext{
${ }^{7}$ Propagation of waves in a nonuniformly moving medium will generally lead to a change of the wave direction; for qualitative estimates we neglect here this effect. This is well justified in the strongly magnetized limit $\sigma \rightarrow \infty$ and/or for small angles $\theta \leq 1$.
} 
then in the laboratory frame the components of the wave velocity along and normal to the bulk velocity are

$$
\beta_{s, \mathrm{lab}, \|}=\frac{\beta+\beta_{s} \cos \theta_{\mathrm{em}}}{1+\beta \beta_{s} \cos \theta_{\mathrm{em}}}, \quad \beta_{s, \mathrm{lab}, \perp}=\frac{\beta_{s} \sin \theta_{\mathrm{em}}}{\Gamma\left(1+\beta \beta_{s} \cos \theta_{\mathrm{em}}\right)} .
$$

The condition for a wave to catch the surface of the shell becomes

$$
\left(R_{\mathrm{em}}+t \beta\right) \sin \theta=\frac{t \beta_{s} \sin \theta_{\mathrm{em}}}{\Gamma\left(1+\beta \beta_{s} \cos \theta_{\mathrm{em}}\right)}, \quad\left(R_{\mathrm{em}}+t \beta\right) \cos \theta=R_{\mathrm{em}}+\frac{\beta+\beta_{s} \cos \theta_{\mathrm{em}}}{1+\beta \beta_{s} \cos \theta_{\mathrm{em}}} t .
$$

Eliminating $\theta_{\mathrm{em}}$ we find

$$
2(\beta \Gamma)^{2}\left(1-\beta_{s}^{2}\right) \sin ^{2} \frac{\theta}{2}=\sqrt{1-\frac{\beta\left(2 R_{\mathrm{em}}+\beta t\right) \beta_{s}^{2}}{\left(R_{\mathrm{em}}+\beta t\right)^{2}}}+\frac{\beta \beta_{s}^{2}}{R_{\mathrm{em}}+\beta t} t-1 .
$$

In the case of an isotropic relativistic fluid with internal sound speed $\beta_{s}=1 / \sqrt{3}$, equation (A3) implies that the maximum angle that sound waves can reach as $t \rightarrow \infty$ is $\theta \sim(\sqrt{6}-2)^{1 / 2} / \gamma \sim 0.67 / \gamma$. Thus, hydrodynamically dominated relativistic plasma remains causally disconnected on scales $\theta \sim 1 / \Gamma$ at all times.

In a magnetically dominated medium the situation is drastically different. Consider, for simplicity, a cold, magnetically dominated plasma. If the ratio of the magnetic to particle energy density in the flow is $\sigma=u_{B} / u_{p} \gg 1$ (Kennel \& Coroniti 1984), the Alfvén velocity is $\beta_{\mathrm{A}}=[\sigma /(1+\sigma)]^{1 / 2}$. Equation (A3) then becomes

$$
2(\beta \Gamma)^{2} \sin ^{2} \frac{\theta}{2}=\sqrt{(1+\sigma)\left[1+\frac{R_{\mathrm{em}}^{2} \sigma}{\left(R_{\mathrm{em}}+\beta t\right)^{2}}\right]}-\left(1+\frac{R_{\mathrm{em}} \sigma}{R_{\mathrm{em}}+\beta t}\right) .
$$

This implies that two points on the surface of the shell separated by an angle $\theta \ll 1$ come into causal contact after a time

$$
\frac{c t}{R_{\mathrm{em}}} \sim \Gamma \theta \sqrt{\frac{1+\sigma}{\sigma}} .
$$

Thus, in a strongly magnetized medium, $\sigma \gg 1$, the visible patch of the shell with $\theta \sim 1 / \Gamma$ reestablishes causal contact in one dynamical time $t \sim R_{\mathrm{em}} / c$.

We can also invert equation (A4) to find the time needed to establish causal contact over an angle $\theta$ :

$t=\frac{2 R_{\mathrm{em}} \beta \Gamma \sin \theta / 2}{\beta\left\{\sigma-4(\beta \Gamma)^{2} \sin ^{2} \theta / 2\left[1+(\beta \Gamma)^{2} \sin ^{2} \theta / 2\right]\right\}}\left(\sqrt{\sigma(1+\sigma)\left[1+(\beta \Gamma)^{2} \sin ^{2} \frac{\theta}{2}\right]}+\beta \Gamma \sin \frac{\theta}{2}\left\{\beta \Gamma+2\left[1+(\beta \Gamma)^{2} \sin ^{2} \frac{\theta}{2}\right]\right\}\right)$.

For $\sigma<4 \Gamma^{2}\left(1+\Gamma^{2}\right)$ the maximum causally connected region (for $t \rightarrow \infty$ ) is finite:

$$
\sin ^{2} \frac{\theta_{\infty}}{2}=\frac{\sqrt{1+\sigma}-1}{2(\beta \Gamma)^{2}}
$$

For a subsonic flow, $\sqrt{\sigma}>\Gamma, \theta_{\infty}$ becomes larger than $1 / \Gamma$. For larger $\sigma$, the whole shell comes into causal contact after a time

$$
t=\frac{2 R_{\mathrm{em}} \beta \Gamma}{\beta\left[\sigma-4 \Gamma^{2}\left(1+\Gamma^{2}\right)\right]}\left\{2(\beta \Gamma)^{3}+\sqrt{\sigma(1+\sigma)\left[1+(\beta \Gamma)^{2}\right]}+\beta \Gamma(2+\sigma)\right\} .
$$

It is also instructive to find the emission angle $\theta_{\mathrm{em}}$ as a function of the time $t$ when an emitted wave catches up with the surface of the shell:

$$
\cos \theta_{\mathrm{em}}=-\frac{1}{\beta \beta_{s}\left(2 R_{\mathrm{em}}+\beta t\right)}\left[\left(R_{\mathrm{em}}+\beta t\right)-\sqrt{\left(R_{\mathrm{em}}+\beta t\right)^{2}-t \beta\left(2 R_{\mathrm{em}}+\beta t\right) \beta_{s}^{2}}\right] .
$$

In an ultramagnetized plasma (force-free plasma, $\sigma \rightarrow \infty$ ), relations simplify considerably. The region causally connected after time $t$ becomes

$$
\sin \frac{\theta}{2}=\frac{1}{2 \Gamma} \sqrt{\frac{t^{2}}{R_{\mathrm{em}}\left(R_{\mathrm{em}}+\beta t\right)}}, \quad t=2 R_{\mathrm{em}} \Gamma \sin \frac{\theta}{2}\left[\beta \Gamma \sin \frac{\theta}{2}+\sqrt{1+(\beta \Gamma)^{2} \sin ^{2} \frac{\theta}{2}}\right],
$$

so that points separated by $1 / \Gamma$ come into causal contact after $t=(1+\sqrt{5}) R_{\mathrm{em}} / 2$, and the whole shell becomes causally connected after $t \sim 2 R_{\mathrm{em}} \Gamma^{2}$. The emission angle (eq. [A9]) in the force-free case then becomes

$$
\cos \theta_{\mathrm{em}}=-\frac{t}{2 R_{\mathrm{em}}+t \beta} .
$$

Note that in the plasma rest frame, the waves that propagate farthest in the polar angle are emitted "backward" in the 
explosion frame. It is due to this fact that they can overcome the common result that the lateral velocity in the laboratory frame cannot be larger than $c / \Gamma$. The latter is true only for waves propagating along the surface of the shell (normal to the flow in the shell frame). We can understand then why hydrodynamic sound waves cannot reach large polar angles: when emitted "backward," they are advected with the supersonically moving flow. On the other hand, in a subsonic, strongly magnetized plasma, fast magnetosound waves can outrun the flow and reach large polar angles in the laboratory frame.

Thus, if the effective signal velocity (Alfvén velocity) in the bulk of the flow is larger than the expansion velocity, strongly relativistic magnetized outflows quickly become causally reconnected over the visible patch $\Delta \theta \sim 1 / \Gamma$. For strongly subsonic flows, $\sigma \gg \Gamma^{2}$, points on the shell separated by $1 / \Gamma$ come into causal contact on a dynamical timescale $t \sim R_{\mathrm{em}}$ (in a relativistic hydrodynamic flow this never happens). Since this time is fairly short, the global dynamics of the flow are not very important, which vindicates our assumption of a constant expansion velocity.

\section{APPENDIX B}

\section{PULSE-INTEGRATED STOKES PARAMETERS}

The Stokes parameters are components of the polarization tensor

$$
J_{l s}=\frac{1}{2}\left(\begin{array}{cc}
I+Q & U \\
U & I-Q
\end{array}\right) .
$$

Here $x_{l}$ are coordinates in the plane perpendicular to $\boldsymbol{n}$, and there is no circular polarization. The pulse-integrated intensity

$$
\overline{\boldsymbol{J}}_{l s}(\nu)=\frac{1}{D^{2}} \int d T \int d V j_{l s}\left(\boldsymbol{n}, \nu, \boldsymbol{r}, T+\frac{r \cos \Theta}{c}\right),
$$

where $j_{l s}$ is the emissivity, $T=t-r \cos \Theta / c$ is the observer time, and the integration is over the whole emitting region in the explosion frame.

We approximate the emissivity as an instant flash at the moment $t=t_{0}$ with the duration $\Delta t \ll \Delta R / c$. We also assume that the whole shell emits uniformly during the flash. Then, the emissivity can be expressed as

$$
j_{l s}(\boldsymbol{n}, \nu, \boldsymbol{r}, t)=j_{l s}\left(\boldsymbol{n}, \nu, t_{0}\right) \delta\left(t-t_{0}\right) \Delta t\left[H\left(r-R_{0}\right)-H\left(r-R_{0}-\Delta R\right)\right],
$$

where $\delta(x)$ is the Dirac delta function and $H(x)$ is a step function: $H(x)=1$ if $x>0$ and $H(x)=0$ if $x<0$. We first integrate over $T$, keeping all other independent variables $(r, \theta, \phi)$ fixed:

$$
\overline{\boldsymbol{J}}_{l s}(\nu)=\frac{1}{D^{2}} \int_{0}^{2 \pi} d \phi \int_{0}^{\pi} \sin \theta d \theta \int_{0}^{+\infty} r^{2} d r j_{l s}\left(\boldsymbol{n}, \nu, \boldsymbol{r}, t_{0}\right) \Delta t\left[H\left(r-R_{0}\right)-H\left(r-R_{0}-\Delta R\right)\right] .
$$

Taking into account that $\Delta R \ll R$ and integrating equation (B3) over $d r$, we obtain

$$
\bar{J}_{l s}(\nu)=\Delta t \Delta R \frac{R_{0}^{2}}{D^{2}} \int_{0}^{2 \pi} d \phi \int_{0}^{\pi} \sin \theta d \theta j_{l s}\left(\boldsymbol{n}, \nu, R_{0}, t_{0}\right) .
$$

The Lorentz transformation of the emissivity to the frame comoving with the element of the shell is

$$
j_{l s}\left(\boldsymbol{n}, \nu, t_{0}\right)=\mathscr{D}^{2}\left(\boldsymbol{n}^{\prime}, t_{0}\right) j_{l{ }^{\prime} s^{\prime}}^{\prime}\left(\boldsymbol{n}^{\prime}, \mathscr{D}^{-1} \nu\right)
$$

so we obtain

$$
\overline{\boldsymbol{J}}_{l s}(\nu)=\Delta t \Delta R \frac{R_{0}^{2}}{D^{2}} \int_{0}^{2 \pi} d \phi \int_{0}^{\pi} \sin \theta d \theta \mathscr{D}^{2+(p-1) / 2} j_{l^{\prime} s^{\prime}}^{\prime}\left(\boldsymbol{n}^{\prime}, \nu\right) .
$$

Using synchrotron expressions for $j_{l^{\prime s^{\prime}}}^{\prime}\left(\boldsymbol{n}^{\prime}, \nu\right)$ in the comoving frame (e.g., Ginzburg 1989), we obtain

$$
\begin{gathered}
\bar{I}=\frac{p+7 / 3}{p+1} \kappa(\nu) \Delta R \Delta t \frac{R_{0}^{2}}{D^{2}(1+z)^{2+(p-1) / 2}} \int_{0}^{2 \pi} d \phi \int_{0}^{\pi} \sin \theta d \theta \mathscr{D}^{2+(p-1) / 2}\left|B^{\prime} \sin \chi^{\prime}\right|^{(p+1) / 2}, \\
\bar{Q}=\kappa(\nu) \Delta R \Delta t \frac{R_{0}^{2}}{D^{2}(1+z)^{2+(p-1) / 2}} \int_{0}^{2 \pi} d \phi \int_{0}^{\pi} \sin \theta d \theta \mathscr{D}^{2+(p-1) / 2}\left|B^{\prime} \sin \chi^{\prime}\right|^{(p+1) / 2} \cos 2 \tilde{\chi}, \\
\bar{U}=\kappa(\nu) \Delta R \Delta t \frac{R_{0}^{2}}{D^{2}(1+z)^{2+(p-1) / 2}} \int_{0}^{2 \pi} d \phi \int_{0}^{\pi} \sin \theta d \theta \mathscr{D}^{2+(p-1) / 2}\left|B^{\prime} \sin \chi^{\prime}\right|^{(p+1) / 2} \sin 2 \tilde{\chi}, \\
\bar{V}=0,
\end{gathered}
$$


where we reinstated the cosmological factor $1+z$. The function $\kappa(\nu)$ is

$$
\kappa(\nu)=\frac{\sqrt{3}}{4} \Gamma_{\mathrm{E}}\left(\frac{3 p-1}{12}\right) \Gamma_{\mathrm{E}}\left(\frac{3 p+7}{12}\right) \frac{e^{3}}{m_{e} c^{2}}\left(\frac{3 e}{2 \pi m_{e}^{3} c^{5}}\right)^{(p-1) / 2} \nu^{-(p-1) / 2} K_{e},
$$

where $e$ and $m_{e}$ are the charge and mass of an electron and $\Gamma_{\mathrm{E}}$ is the Euler gamma function.

The degree of polarization of the observed radiation pulse is expressed as $\Pi=\left(\bar{Q}^{2}+\bar{U}^{2}\right)^{1 / 2} / \bar{I}$, giving equation $(2)$. The resulting position angle of the electric field $\tilde{\chi}_{\text {res }}$ measured by the observer is found from

$$
\cos 2 \tilde{\chi}_{\mathrm{res}}=\frac{\bar{Q}}{\sqrt{\bar{Q}^{2}+\bar{U}^{2}}}, \quad \sin 2 \tilde{\chi}_{\mathrm{res}}=\frac{\bar{U}}{\sqrt{\bar{Q}^{2}+\bar{U}^{2}}}, \quad 0 \leq \tilde{\chi}_{\mathrm{res}}<\pi .
$$

It can be checked that in our case, under the change of $\phi$ to $2 \pi-\phi$ in the integrals in equations (B7), the value of $\bar{Q}$ is not changed and the sign of $\bar{U}$ is reversed. Therefore, the Stokes parameter $\bar{U}$ integrates out to zero. Consequently, if $\bar{Q}>0$, then $\tilde{\chi}_{\text {res }}=0$, and if $\bar{Q}<0$, then $\tilde{\chi}_{\text {res }}=\pi / 2$. Thus, the observed electric vector can be either parallel or perpendicular to the projection of the axis of the flow on the plane of the sky. For a shell carrying only a toroidal magnetic field, $\bar{Q}>0$.

\section{APPENDIX C}

\section{LORENTZ TRANSFORMATIONS OF THE POLARIZATION VECTOR}

In this appendix we first derive the Lorentz transformations of the polarization vector of the linearly polarized radiation emitted by a relativistically moving plasma with a given magnetic field and then find the angle $\tilde{\chi}$ between a given direction (chosen later as the direction along the projection of the axis of the flow in the plane of the sky) and the direction of linear polarization of the waves for a spherically expanding shell.

Let $\boldsymbol{n}^{\prime}$ be a unit vector in the direction of a wavevector in the plasma rest frame and $\hat{\boldsymbol{B}}^{\prime}$ be a unit vector along the magnetic field in the plasma rest frame. The electric field of a linearly polarized electromagnetic wave is directed along the unit vector $\hat{\boldsymbol{e}}^{\prime}=\boldsymbol{n}^{\prime} \times \hat{\boldsymbol{B}}^{\prime}$, and the magnetic field of the wave is along the unit vector $\hat{\boldsymbol{b}}^{\prime}=\boldsymbol{n}^{\prime} \times \hat{\boldsymbol{e}}^{\prime}$, such that the Poynting flux along $\hat{\boldsymbol{e}}^{\prime} \times \hat{\boldsymbol{b}}^{\prime}$ is directed along $\boldsymbol{n}^{\prime}$. We give a Lorentz boost to the explosion frame to find the electric field $\boldsymbol{e}$ there, normalize it to unity, and project $\boldsymbol{e}$ on some given direction (e.g., along the projection of the flow axis on the plane of the sky).

Fields in the wave expressed in terms of the direction of a photon in the explosion frame $\boldsymbol{n}$ are

$$
\begin{gathered}
\hat{\boldsymbol{e}}^{\prime}=\frac{\boldsymbol{n} \times \hat{\boldsymbol{B}}^{\prime}}{\Gamma(1-\boldsymbol{n} \cdot \boldsymbol{v})}+\frac{1+\Gamma(1-\boldsymbol{n} \cdot \boldsymbol{v})}{(1+\Gamma)(1-\boldsymbol{n} \cdot \boldsymbol{v})} \hat{\boldsymbol{B}}^{\prime} \times \boldsymbol{v}, \\
\hat{\boldsymbol{b}}^{\prime}=-\hat{\boldsymbol{B}}^{\prime}+\left[\frac{\hat{\boldsymbol{B}}^{\prime} \cdot \boldsymbol{n}}{\Gamma^{2}(1-\boldsymbol{n} \cdot \boldsymbol{v})^{2}}-\frac{1+\Gamma(1-\boldsymbol{n} \cdot \boldsymbol{v})}{\Gamma(1+\Gamma)(1-\boldsymbol{n} \cdot \boldsymbol{v})^{2}} \hat{\boldsymbol{B}}^{\prime} \cdot \boldsymbol{v}\right] \boldsymbol{n}+\left\{\frac{[1+\Gamma(1-\boldsymbol{n} \cdot \boldsymbol{v})]^{2}}{(1+\Gamma)^{2}(1-\boldsymbol{n} \cdot \boldsymbol{v})^{2}} \hat{\boldsymbol{B}}^{\prime} \cdot \boldsymbol{v}-\frac{1+\Gamma(1-\boldsymbol{n} \cdot \boldsymbol{v})}{\Gamma(1+\Gamma)(1-\boldsymbol{n} \cdot \boldsymbol{v})^{2}} \hat{\boldsymbol{B}}^{\prime} \cdot \boldsymbol{n}\right\} \boldsymbol{v} .
\end{gathered}
$$

It can be verified that $\hat{\boldsymbol{e}}^{\prime} \times \hat{\boldsymbol{b}}^{\prime}$ is still directed along $\boldsymbol{n}^{\prime}$.

Next, we make a Lorentz transformation of $\hat{\boldsymbol{e}}^{\prime}$ back to the lab frame,

$$
\boldsymbol{e}=\Gamma\left[\hat{\boldsymbol{e}}^{\prime}-\frac{\Gamma}{\Gamma+1}\left(\hat{\boldsymbol{e}}^{\prime} \cdot \boldsymbol{v}\right) \boldsymbol{v}-\boldsymbol{v} \times \hat{\boldsymbol{b}}^{\prime}\right],
$$

and normalize to unity. After some rearrangement, we find

$$
\hat{\boldsymbol{e}}=\frac{\boldsymbol{n} \times \boldsymbol{q}^{\prime}}{\sqrt{q^{\prime 2}-\left(\boldsymbol{n} \cdot \boldsymbol{q}^{\prime}\right)^{2}}}, \quad \boldsymbol{q}^{\prime}=\hat{\boldsymbol{B}}^{\prime}+\boldsymbol{n} \times\left(\boldsymbol{v} \times \hat{\boldsymbol{B}}^{\prime}\right)-\frac{\Gamma}{1+\Gamma}\left(\hat{\boldsymbol{B}}^{\prime} \cdot \boldsymbol{v}\right) \boldsymbol{v} .
$$

Finally, we can express the rest-frame unit vector $\hat{\boldsymbol{B}}^{\prime}$ in terms of the laboratory-frame unit vector $\hat{\boldsymbol{B}}$. Assuming ideal MHD, there is no electric field in the rest frame of the plasma: $\boldsymbol{E}^{\prime}=0$. Then, we obtain

$$
\hat{\boldsymbol{B}}=\frac{1}{\sqrt{1-\left(\hat{\boldsymbol{B}}^{\prime} \cdot \boldsymbol{v}\right)^{2}}}\left[\hat{\boldsymbol{B}}^{\prime}-\frac{\Gamma}{1+\Gamma}\left(\hat{\boldsymbol{B}}^{\prime} \cdot \boldsymbol{v}\right) \boldsymbol{v}\right], \quad \hat{\boldsymbol{B}}^{\prime}=\frac{(1+\Gamma) \hat{\boldsymbol{B}}+\Gamma^{2}(\hat{\boldsymbol{B}} \cdot \boldsymbol{v}) \boldsymbol{v}}{(1+\Gamma) \sqrt{1+\Gamma^{2}(\hat{\boldsymbol{B}} \cdot \boldsymbol{v})^{2}}},
$$

to get

$$
\hat{\boldsymbol{e}}=\frac{\boldsymbol{n} \times \boldsymbol{q}}{\sqrt{q^{2}-(\boldsymbol{n} \cdot \boldsymbol{q})^{2}}}, \quad \boldsymbol{q}=\hat{\boldsymbol{B}}+\boldsymbol{n} \times(\boldsymbol{v} \times \hat{\boldsymbol{B}}) .
$$

This is a general expression giving the polarization vector in terms of the observed quantities $\hat{\boldsymbol{B}}, \boldsymbol{n}$, and $\boldsymbol{v}$. If, for a moment, we 


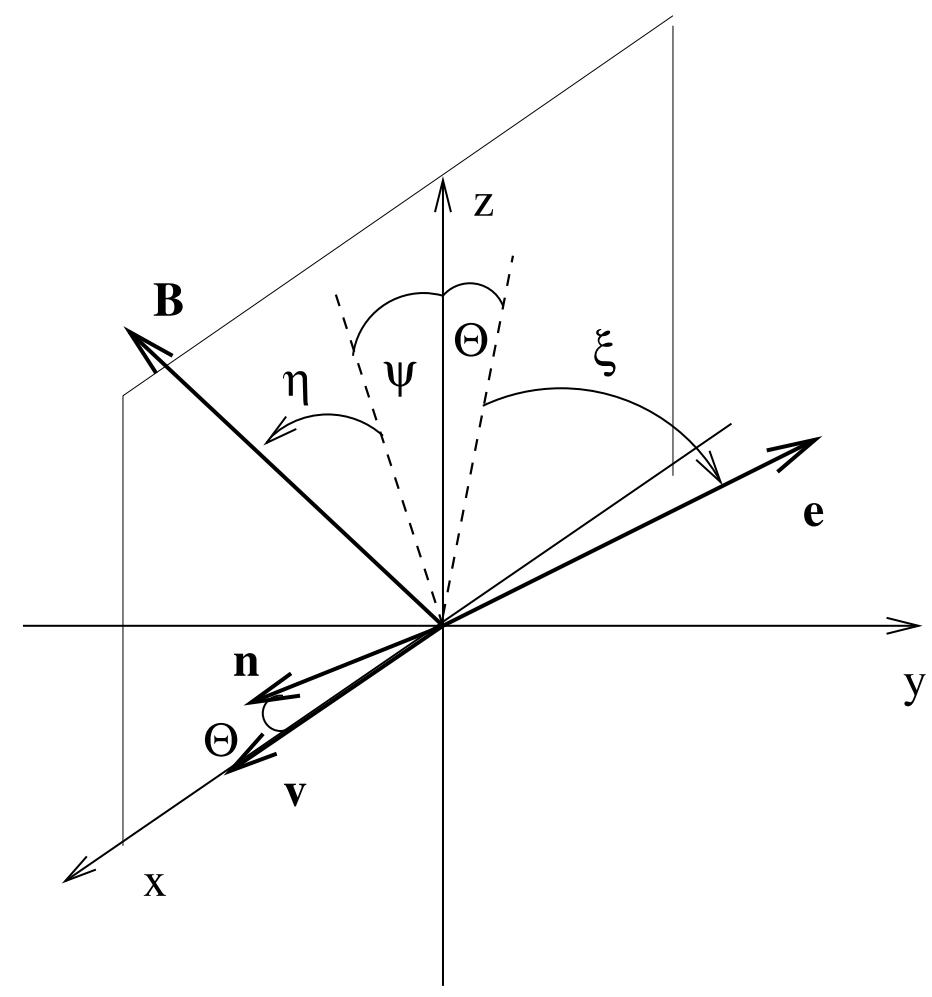

FIG. 4.-Characteristic swing of the polarization angle due to relativistic motion. In the frame aligned with $\boldsymbol{v}$, the electric field of the wave $\boldsymbol{e}$ and the observed magnetic field $\boldsymbol{B}$ make angles $\xi$ and $\eta$ with the plane containing $\boldsymbol{v}$ and $\boldsymbol{n}$, while their projections make angles $\Theta$ and $\psi$ with the $z$-axis perpendicular to the $\boldsymbol{v}$ - $\boldsymbol{n}$ plane (after Blandford \& Königl 1979).

adopt a frame aligned with the direction of motion (Fig. 4), we find from equation (C5)

$$
\tan \xi=\cot \eta \frac{\cos (\Theta+\psi)-\beta \cos \psi}{1-\beta \cos \Theta}
$$

reproducing equation (16) in Blandford \& Königl (1979).

In our particular case $\hat{\boldsymbol{B}}^{\prime} \cdot \boldsymbol{v}=0$, so that the fields in the rest frame of the emitting plasma element and the laboratory frame are aligned: $\hat{\boldsymbol{B}}^{\prime}=\hat{\boldsymbol{B}}$. The general relations then simplify. Setting $\hat{\boldsymbol{B}}^{\prime} \cdot \boldsymbol{v}=0$ in equation (C3) gives

$$
\hat{\boldsymbol{e}}=\frac{\boldsymbol{n} \times\{\hat{\boldsymbol{B}}+[\boldsymbol{n} \times(\boldsymbol{v} \times \hat{\boldsymbol{B}})]\}}{\sqrt{(1-\boldsymbol{n} \cdot \boldsymbol{v})^{2}-(\hat{\boldsymbol{B}} \cdot \boldsymbol{n})^{2} / \Gamma^{2}}} .
$$

Next, we introduce a unit vector $\boldsymbol{l}$ normal to the plane containing $\boldsymbol{n}$ and some given direction (in our case the direction of the projection of the axis of the flow to the plane of the sky). Then,

$$
\cos \tilde{\chi}=\hat{\boldsymbol{e}} \cdot(\boldsymbol{n} \times \boldsymbol{l}), \quad \sin \tilde{\chi}=\hat{\boldsymbol{e}} \cdot \boldsymbol{l} .
$$

Using equation (C7) we find

$$
\begin{gathered}
\cos \tilde{\chi}=\frac{1}{\sqrt{1-\{\hat{\boldsymbol{B}} \cdot \boldsymbol{n} /[\Gamma(1-\boldsymbol{n} \cdot \boldsymbol{v})]\}^{2}}}\left\{\hat{\boldsymbol{B}} \cdot\left[\boldsymbol{l}+\frac{(\boldsymbol{l} \cdot \boldsymbol{v})}{1-\boldsymbol{n} \cdot \boldsymbol{v}} \boldsymbol{n}\right]\right\}, \\
\sin \tilde{\chi}=\frac{\Gamma \quad\left[(\hat{\boldsymbol{B}} \cdot \boldsymbol{l} \times \boldsymbol{n})+\frac{\Gamma(\boldsymbol{n} \cdot \boldsymbol{v})(\hat{\boldsymbol{B}} \cdot \boldsymbol{v} \times \boldsymbol{l})}{(1+\Gamma)}+\frac{\Gamma(\hat{\boldsymbol{B}} \cdot \boldsymbol{n} \times \boldsymbol{v})(\boldsymbol{l} \cdot \boldsymbol{v})}{(1+\Gamma)}+\frac{(\hat{\boldsymbol{B}} \cdot \boldsymbol{n})(\boldsymbol{l} \cdot \boldsymbol{n} \times \boldsymbol{v})}{\Gamma(1-\boldsymbol{n} \cdot \boldsymbol{v})}\right] .}{\sqrt{1-\left\{\hat{\boldsymbol{B}} \cdot \boldsymbol{n} /[\Gamma(1-\boldsymbol{n} \cdot \boldsymbol{v})\}^{2}\right.}} .
\end{gathered}
$$

In our case,

$$
\begin{gathered}
\hat{\boldsymbol{B}}=\{-\sin \phi, \cos \phi, 0\}, \quad \boldsymbol{n}=\left\{\sin \theta_{\mathrm{ob}}, 0, \cos \theta_{\mathrm{ob}}\right\}, \quad \boldsymbol{v}=\beta\{\sin \theta \cos \phi, \sin \theta \sin \phi, \cos \theta\}, \\
\boldsymbol{l}=\{0,1,0\}, \quad \boldsymbol{n} \cdot \boldsymbol{v}=\beta \mu, \quad \mu=\cos \Theta=\cos \theta \cos \theta_{\mathrm{ob}}+\sin \theta \sin \theta_{\mathrm{ob}} \cos \phi,
\end{gathered}
$$

which gives equation (7). 


\section{APPENDIX D}

\section{THE ULTRARELATIVISTIC LIMIT}

In the ultrarelativistic limit, when $\Gamma \gg 1$, the maximum polarization fraction for a given velocity field can be found analytically. Because of the Doppler boosting effect described by the factor $\mathscr{D}$, the contribution to the integrals in formula (2) comes from the small patch $\phi \sim 1 / \Gamma$ and $\theta-\theta_{\mathrm{ob}} \sim 1 / \Gamma$. We can introduce rescaled variables $\xi=\Gamma \phi$ and $\Psi=\Gamma\left(\theta-\theta_{\mathrm{ob}}\right)$ and change the integration from $\theta$ and $\phi$ to $\xi$ and $\Psi$. The integration limits can be taken from $-\infty$ to $+\infty$ for both $\xi$ and $\Psi$. Making expansions for $\Gamma \gg 1, \xi \sim O(1)$, and $\Psi \sim O(1)$, expression (7) results in

$$
\cos 2 \tilde{\chi}=\frac{\left(\Psi^{2}-\xi^{2} \sin ^{2} \theta_{\mathrm{ob}}+1\right)^{2}-4 \Psi^{2} \xi^{2} \sin ^{2} \theta_{\mathrm{ob}}}{\left(\Psi^{2}-\xi^{2} \sin ^{2} \theta_{\mathrm{ob}}+1\right)^{2}+4 \Psi^{2} \xi^{2} \sin ^{2} \theta_{\mathrm{ob}}}
$$

the expression for $\sin \chi^{\prime}$ becomes

$$
\sin ^{2} \chi^{\prime}=1-\frac{4 \xi^{2} \sin ^{2} \theta_{\mathrm{ob}}}{\left(1+\Psi^{2}+\xi^{2} \sin ^{2} \theta_{\mathrm{ob}}\right)^{2}}
$$

and the expression for $\mathscr{D}$ becomes

$$
\mathscr{D}=\frac{2 \Gamma}{\Psi^{2}+\xi^{2} \sin ^{2} \theta_{\mathrm{ob}}+1} .
$$

Note that the variables $\xi$ and $\sin \theta_{\mathrm{ob}}$ enter in the integrals in equation (2) only in the combination $\xi \sin \theta_{\mathrm{ob}}$. Therefore, by changing the integration to a new variable $\xi_{1}=\xi \sin \theta_{\mathrm{ob}}$, the values of the integrals and $\Pi$ become independent of $\theta_{\mathrm{ob}}$. Therefore, for $\Gamma \gg 1$ the polarization degree is insensitive to $\theta_{\mathrm{ob}}$, as long as $\theta_{\mathrm{ob}} \gg 1 / \Gamma$ (see Fig. 3).

Further, it is convenient to switch to the integration in "polar" coordinates $\sigma$ and $\tau$ in the plane of $\xi_{1}$ and $\Psi$, which are introduced according to $\sigma=\xi_{1}^{2}+\Psi^{2}, \xi_{1}=\sqrt{\sigma} \cos (\tau / 2)$, and $\Psi=\sqrt{\sigma} \sin (\tau / 2)$. After some algebra we obtain

$$
\Pi=\frac{p+1}{p+7 / 3} \frac{\int_{0}^{\infty} d \sigma \int_{0}^{2 \pi} d \tau\left(1+\sigma^{2} \cos 2 \tau-2 \sigma \cos \tau\right)\left(1+\sigma^{2}-2 \sigma \cos \tau\right)^{(p-3) / 4} /(1+\sigma)^{2+p}}{\int_{0}^{\infty} d \sigma \int_{0}^{2 \pi} d \tau\left(1+\sigma^{2}-2 \sigma \cos \tau\right)^{(p+1) / 4} /(1+\sigma)^{2+p}} .
$$

For $p=3$ expression (D4) gives $\Pi=9 / 16 \approx 56 \%$. This value is the value of the horizontal asymptotic of the $\Gamma\left(\theta_{\mathrm{ob}}\right)$ curve for $p=3$ in Figure 3. For arbitrary $p$, asymptotic values of the polarization are plotted in Figure 5.

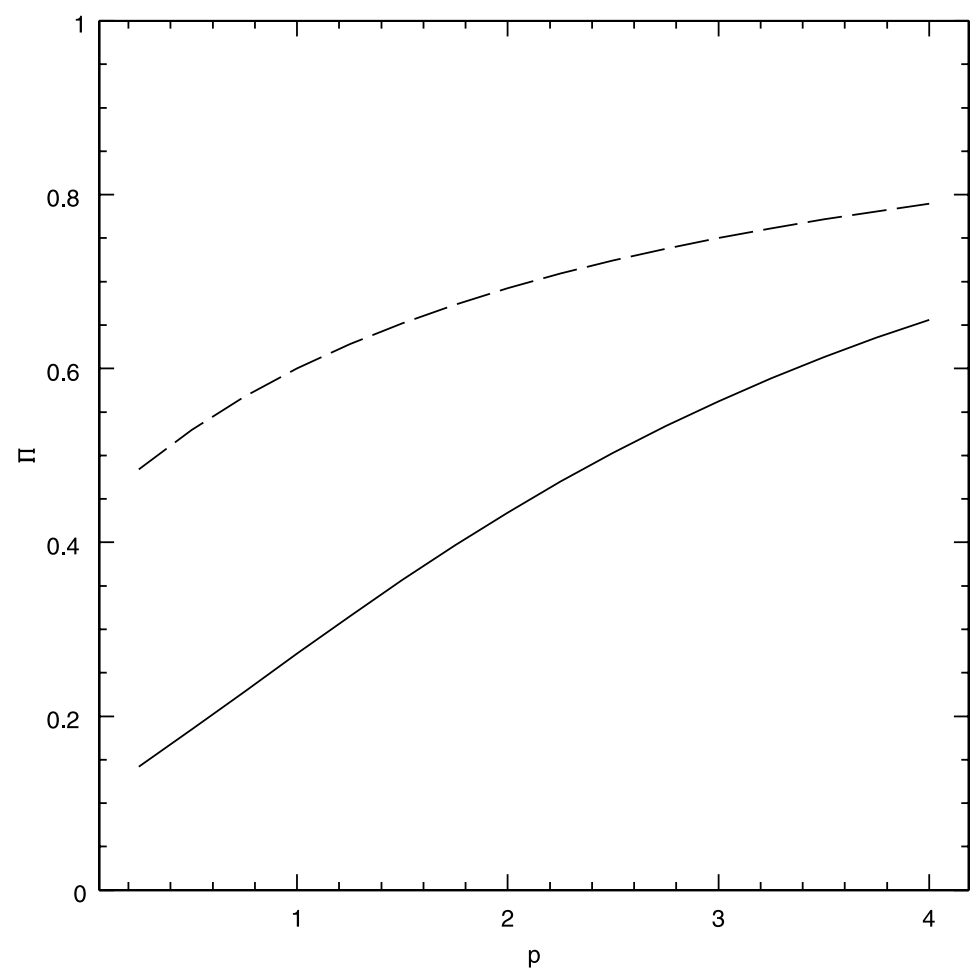

Fig. 5.-Asymptotic value of the polarization for $\Gamma \rightarrow \infty$ as a function of $p$ for a spherically divergent flow (solid line) and the maximum polarization $\Pi_{\text {max }}$ for a homogeneous stationary magnetic field (dashed line). 
Barth, A. J., et al. 2003, ApJ, 584, L47

Bersier, D., et al. 2003, ApJ, 583, L63

Bjornsson, C.-I. 1982, ApJ, 260, 855

Blandford, R. D. 2002, in Lighthouses of the Universe: The Most

Luminous Celestial Objects and Their Use for Cosmology, ed.

M. Gilfanov, R. A. Siuniaev, \& E. Churazov (Berlin: Springer), 381

Blandford, R. D., \& Königl, A. 1979, ApJ, 232, 34

Coburn, W., \& Boggs, S. E. 2003, Nature, 423, 415

Cocke, W. J., \& Holm, D. A. 1972, Nature Phys. Sci., 240, 161

Covino, S., Ghisellini, G., Lazzati, D., \& Malesani, D. 2003a, in Proc. Gamma-Ray Bursts in the Afterglow Era: 3d Workshop (astro-ph/ 0301608)

Covino, S., et al. 2003b, A\&A, 400, L9

Dar, A. 2003, in Proc. Gamma-Ray Bursts in the Afterglow Era: 3d Workshop (astro-ph/0301389)

Dermer, C. D. 2002, ApJ, 574, 65

Drenkhahn, G., \& Spruit, H. C. 2002, A\&A, 391, 1141

Ginzburg, V. L. 1989, Applications of Electrodynamics in Theoretical Physics and Astrophysics. (New York: Gordon \& Breach)

Goldreich, P., \& Julian, W. H. 1969, ApJ, 157, 869

Granot, J. 2003, ApJ, 596, L17

Gruzinov, A., \& Waxman, E. 1999, ApJ, 511, 852

Inoue, S., Guetta, D., \& Pacini, F. 2003, ApJ, 583, 379

Kennel, C. F., \& Coroniti, F. V. 1984, ApJ, 283, 694

\section{REFERENCES}

Königl, A., \& Granot, J. 2002, ApJ, 574,134

Korchakov, A. A., \& Syrovatskii, S. I. 1962, Soviet Astron., 5, 678

Lyutikov, M. 2002, Phys. Fluids, 14, 963

Lyutikov, M., \& Blandford, R. D. 2002, in Beaming and Jets in Gamma-Ray Bursts, ed. R. Ouyed, J. Hjorth, \& A. Nordlund, in press

McComas, D. J., et al. 2000, J. Geophys. Res., 105, 10419

Medvedev, M. V., \& Loeb, A. 1999, ApJ, 526, 697

Mészáros, P. 2002, ARA\&A, 40, 137

Mészáros, P., \& Rees, M. J. 1997, ApJ, 482, L29

Parker, E. N. 1960, ApJ, 132, 821

Piran, T. 1999, Phys. Rep., 314, 575

Rol, E., et al. 2003, A\&A, 405, L23

Rossi, E., Lazzati, D., Salmonson, J. D., \& Ghisellini, G. 2002, in Beaming and Jets in Gamma-Ray Bursts, ed. R. Ouyed, J. Hjorth, \& A. Nordlund, in press

Sari, R. 1999, ApJ, 524, L43

Smolsky, M. V., \& Usov, V. V. 1996, ApJ, 461, 858

Spruit, H. C., Daigne, F., \& Drenkhahn, G. 2001, A\&A, 369, 694

Thompson, A. C. 1994, MNRAS, 270, 480

Usov, V. V. 1992, Nature, 357, 472

Vietri, M., \& Stella, L. 1999, ApJ, 527, L43

Vlahakis, N., \& Königl, A. 2003, ApJ, 596, 1104

Waxman, E. 2003, Nature, 423, 388 